Textures and Microstructures, 1988, Vol. 7, pp. 231-263

Reprints available directly from the publisher

Photocopying permitted by license only

(C) 1988 Gordon and Breach Science Publishers Inc.

Printed in the United Kingdom

\title{
Orientation Correlation in Grain and Phase Boundaries
}

\author{
H. J. BUNGE and H. WEILAND \\ Department of Physical Metallurgy Technical University of Clausthal, FRG
}

(Received September 4, 1987)

The properties of grain and phase boundaries depend on five angular coordinates, i.e. three parameters specifying the orientation difference across the boundary and two parameters specifying the orientation of the boundary normal direction in space or with respect to the crystal lattice. Hence, five-dimensional boundary distribution functions have to be considered. If one considers only misorientation a threedimensional misorientation distribution function is obtained. The deviation of this function from the "uncorrelated" misorientation distribution yields the orientation correlation function. The most economical representation of these functions is the one using series expansions in terms of symmetrized harmonic functions. With the present state of experimental technique it seems to be impossible to determine the complete boundary distribution functions. However, two-dimensional analoga of these functions can be obtained from electron diffraction measurements.

KEY WORDS: Grain boundaries, Phase boundaries, Five-dimensional boundary distribution, Misorientation distribution, Orientation correlation, Series expansion, Electron diffraction, Dual phase steel.

\section{GENERAL DEFINITIONS}

In a polycrystalline polyphase material consisting of $n$ different phases, $n^{2}$ types of interphase boundaries may occur (including grain boundaries between grains of equal phases), Figure 1. A boundary between two crystals (of the same phase or different phases) is characterized by the orientation difference of the two adjacent crystallites and the normal direction of the boundary itself, Figure 2. The considered grain of the phase $\alpha$ may have the orientation $g_{\alpha}$ (with respect to a sample fixed coordinate system $K_{A}$ ) 


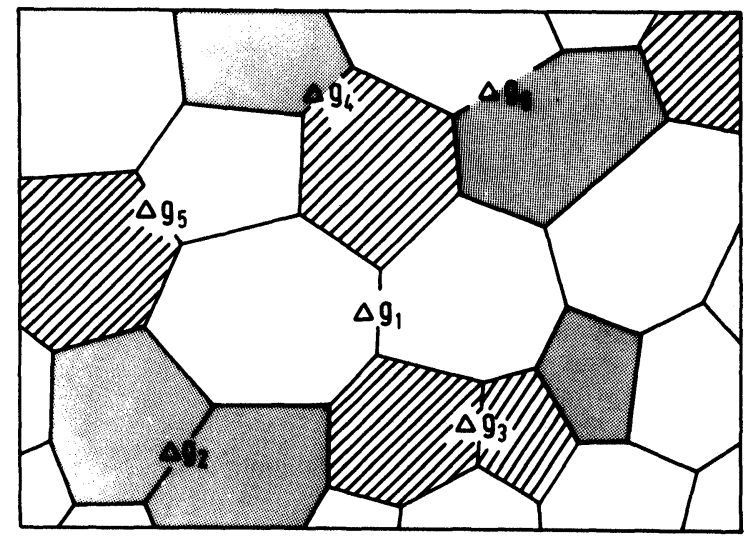

Figure 1 Grain and phase boundaries in a polyphase material.

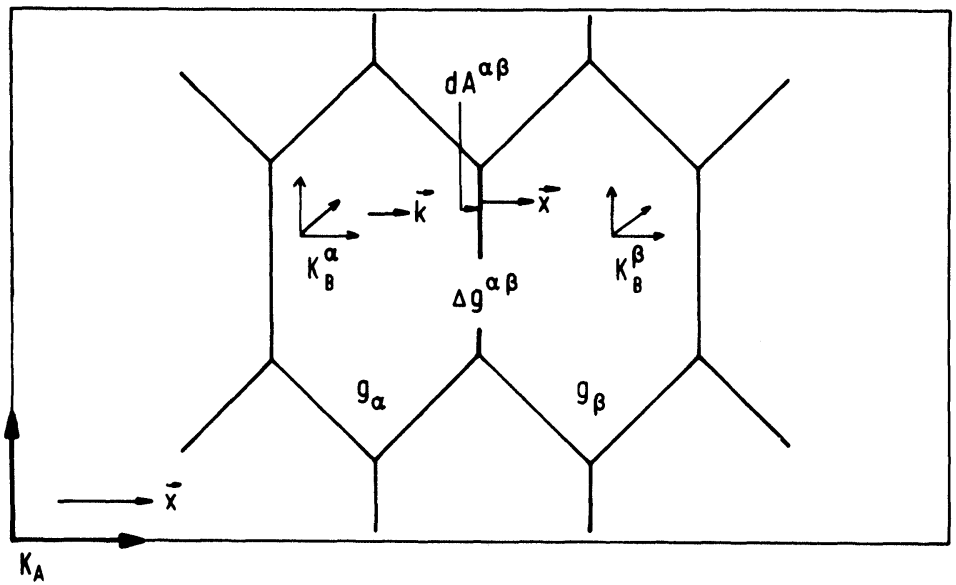

Figure 2 Definition of the sample coordinate system $K_{A}$ and the crystal coordinate systems $K_{B}^{\alpha}$ and $K_{B}^{\beta}$ in the grains of the orientations $g_{\alpha}$ and $g_{\beta}$ of the phases $\alpha$ and $\beta$ respectively. The orientation difference across the phase boundary is $\Delta g_{\alpha \beta}$. The area of this boundary is $d A^{\alpha \beta}$. The normal direction of the phase boundary is described by the vector $\mathbf{x}$ in the sample coordinate system or by the vector $k$ in the crystal coordinate system of the $\alpha$-phase. 
that of the phase $\beta$ the orientation $g_{\beta}$. Then the orientation difference across the boundary is defined as the rotation $\Delta g_{\alpha \beta}$ which transforms the crystal coordinate system $K_{B}^{\alpha}$ of the first crystal into that of the second one, $K_{B}^{\beta}$. The orientation difference is thus defined by

$$
\Delta g_{\alpha \beta}=g_{\beta} \cdot g_{a}^{-1}
$$

It may be specified in several different ways of which the transformation matrix $\left[a_{i k}\right]$, rotation axis and angle $\left\{d_{{ }_{\gamma}}, \omega\right\}$, or the Euler angles $\left\{\varphi_{1}, \phi, \varphi_{2}\right\}$ are the most frequently used ones (see e.g. Bunge 1982)

$$
\Delta g_{\alpha \beta}=\left\{a_{i k}\right\}=\left\{d_{\theta \psi}, \omega\right\}=\left\{\varphi_{1}, \phi, \varphi_{2}\right\}
$$

The normal direction of a boundary element may be specified by a unit vector $\mathrm{x}$ pointing in the direction from the phase $\alpha$ towards $\beta$. Alternatively, if the crystallographic nature of the phase boundry is to be considered rather than its orientation in space, the normal direction of the boundary may be referred to the crystal coordinate system $K_{B}^{\alpha}$. The boundary normal is then a specific crystal direction $\mathbf{k}$ of the $\alpha$ crystal. The vectors $\mathbf{x}$ and $\mathbf{k}$ may be specified by cartesian coordinates or by spherical polar coordinates respectively in the coordinate system $K_{A}$ and $K_{B}^{\alpha}$

$$
\begin{aligned}
& \mathbf{x}=\left\{x_{1}, x_{2}, x_{3}\right\}_{K_{A}}=\{\alpha, \beta\}_{K_{A}} \\
& \mathbf{k}=\left\{k_{1}, k_{2}, k_{3}\right\}_{K_{B}^{\alpha}}=\{\theta, \gamma\}_{K_{B}^{\alpha}}
\end{aligned}
$$

(Thereby the angles $\alpha \beta$ must not be mixed up with the phase indices $\alpha \beta$ ). The two vectors $\mathbf{x}$ and $\mathbf{k}$ are related to each other by the orientation $g_{\alpha}$ of the $\alpha$ crystal

$$
\mathbf{x}=g_{\alpha} \cdot \mathbf{k}
$$

We consider the area fraction of $\alpha \beta$-boundaries which are characterized by the orientation difference $\Delta g$ within the limits $d \Delta g$ and the surface normal direction $\mathbf{x}$ or $\mathbf{k}$ within the solid angular range $d \Omega$. We thus obtain two distribution functions

$$
\begin{aligned}
& \frac{d A^{\alpha \beta}(\Delta g, \mathbf{x})}{A^{\alpha \beta}}=\phi^{\alpha \beta}(\Delta g, \mathbf{x}) d \Delta g d \Omega \\
& \frac{d A^{\alpha \beta}(\Delta g, \mathbf{k})}{A^{\alpha \beta}}=\psi^{\alpha \beta}(\Delta g, \mathbf{k}) d \Delta g d \Omega
\end{aligned}
$$


whereby $A^{\alpha \beta}$ is the total area of $\alpha \beta$-boundaries in the sample. The element $d \Delta g$ in the misorientation space $\Delta g$ is defined in such a way that

$$
\int d \Delta g=1
$$

Expressed in Euler angles, the element $d \Delta g$ is then to be written

$$
d \Delta g=\frac{\sin \phi}{8 \pi^{2}} d \varphi_{1} d \phi d \varphi_{2}
$$

Similarly we assume

$$
\int d \Omega=1
$$

which requires, in terms of spherical polar coordinates,

$$
d \Omega=\frac{\sin \alpha}{4 \pi} d \alpha d \beta
$$

With these definitions, the functions $\phi$ and $\psi$ are normalized in multiples of the random distribution, i.e. it is

$$
\phi_{\text {rand }}^{\alpha \beta}(\Delta g, \mathbf{x})=\psi_{\text {rand }}^{\alpha \beta}(\Delta g, \mathbf{k}) \equiv 1
$$

The distribution functions $\phi^{\alpha \beta}$ and $\psi^{\alpha \beta}$ are, in principle, independent of each other.

Three different integrals of these functions may be considered. Integration over the boundary normal orientations (in both coordinate systems) yields the physical misorientation distribution function

$$
F^{\alpha \beta}(\Delta g)=\int \phi^{\alpha \beta}(\Delta g, \mathbf{x}) d \Omega=\int \psi^{\alpha \beta}(\Delta g, \mathbf{k}) d \Omega
$$

which is defined by the area fraction of boundaries across which the orientation difference or "misorientation" $\Delta g$ occurs (Ref. Bunge 1982)

$$
\frac{d A^{\alpha \beta}(\Delta g)}{A^{\alpha \beta}}=F^{\alpha \beta}(\Delta g) d \Delta g
$$

Integration over the orientation difference $\Delta g$ yields the two "shape 
functions" (Hilliard 1982, Bunge 1969)

$$
\begin{array}{r}
S^{\alpha \beta}(\mathbf{x})=\int \phi^{\alpha \beta}(\Delta g, \mathbf{x}) d \Delta g \\
\sigma^{\alpha \beta}(\mathbf{k})=\int \psi^{\alpha \beta}(\Delta g, \mathbf{k}) d \Delta g
\end{array}
$$

They describe the orientation of the boundaries in space $\mathbf{x}$ or with respect to the crystallographic nature $\mathbf{k}$ (habit plane). In single phase materials, where only grain boundaries $\alpha \alpha$ occur, these two functions describe the grain shape in space and the grain shape with respect to the crystallographic nature i.e. the "habitus" of the grain. From Eq. (11) it is seen that the distribution functions $\phi^{\alpha \beta}$ and $\psi^{\alpha \beta}$ are not completely independent of each other.

It is further to be mentioned, that the boundary distribution functions $\phi^{\alpha \beta}$ and $\psi^{\alpha \beta}$ are, in principle, independent of the textures of the phases, i.e. the orientation distributions of the volume fractions of the phases, which are defined by

$$
\frac{d V^{\alpha}(g)}{V^{\alpha}}=f^{\alpha}(g) d g
$$

Where $V^{\alpha}$ is the volume of the phase $\alpha$ in the polyphase material irrespective of crystal orientation.

Interchanging the phases $\alpha$ and $\beta$ leads to

Furthermore, it is

$$
\Delta g_{\beta \alpha}=g_{\alpha} \cdot g_{\beta}^{-1}=\Delta g_{\alpha \beta}^{-1}
$$

and

$$
\mathbf{x}_{\beta}=-\mathbf{x}_{\alpha}
$$

$$
\mathbf{k}_{\beta}=-\Delta g_{\alpha \beta}^{-1} \cdot \mathbf{k}_{\alpha}
$$

Hence, the corresponding distribution functions obey the interchange relations

$$
\begin{gathered}
\phi^{\beta \alpha}(\Delta g, \mathbf{x})=\phi^{\alpha \beta}\left(\Delta g^{-1},-\mathbf{x}\right) \\
\psi^{\beta \alpha}(\Delta g, \mathbf{k})=\psi^{\alpha \beta}\left(\Delta g^{-1},-\Delta g \cdot \mathbf{k}\right) \\
F^{\beta \alpha}(\Delta g)=F^{\alpha \beta}\left(\Delta g^{-1}\right) \\
S^{\beta \alpha}(\mathbf{x})=S^{\alpha \beta}(-\mathbf{x})
\end{gathered}
$$

These equations are inter-functional relations in the case of phase 
boundaries and they are intra-functional relations for the grain boundary distribution functions.

\section{SYMMETRIES}

Two types of symmetries are to be considered in a polycrystalline material. These are the crystal symmetry and the sample symmetry. In a polyphase material, each phase may have its own crystal symmetry. It must, however, be assumed that there is only one sample symmetry (Bunge 1985). The symmetries are characterized by point symmetry groups $G$ containing symmetry operations $o$ which may be rotations $g$ or inversion $u$.

$$
\begin{aligned}
& G^{s}=\left\{o^{s}\right\}=\left\{g^{s}, u^{s}\right\} \quad \text { sample symmetry } \\
& G^{c_{\alpha}}=\left\{o^{c_{\alpha}}\right\}=\left\{g^{c_{\alpha}}, u^{c_{\alpha}}\right\} \text { crystal symmetry phase } \alpha
\end{aligned}
$$

Directions (sample or crystal) are equivalent to each other by the full point symmetry group which is the crystal symmetry for the crystal directions $\mathbf{k}$ and the sample symmetry for the sample directions $\mathbf{x}$. Hence, symmetrical equivalence of the directions $\mathbf{x}$ and $\mathbf{x}^{\prime}$ and $\mathbf{k}$ and $\mathbf{k}^{\prime}$ is defined by

$$
\begin{aligned}
\mathbf{x}^{\prime} \leftarrow \text { equiv } \rightarrow \mathbf{x} & \text { where } \quad \mathbf{x}^{\prime}=o^{s} \cdot \mathbf{x} \\
\mathbf{k}^{\prime} \leftarrow \text { equiv } \rightarrow \mathbf{k} & \text { where } \quad \mathbf{k}^{\prime}=o^{c} \cdot \mathbf{k}
\end{aligned}
$$

As far as orientations and orientation differences are concerned, only the rotational elements of the symmetry groups are to be considered (Bunge, Esling 1985). Hence, symmetrically equivalent orientations or orientation differences are defined

$$
\begin{array}{rcc}
g_{\alpha}^{\prime} \leftarrow \text { equiv } \rightarrow g_{\alpha} & \text { where } & g_{\alpha}^{\prime}=g^{c_{\alpha}} \cdot g_{\alpha} \cdot g^{s} \\
\Delta g_{\alpha \beta}^{\prime} \leftarrow \text { equiv } \rightarrow \Delta g_{\alpha \beta} & \text { where } & \Delta g_{\alpha \beta}^{\prime}=g^{c_{\beta}} \cdot \Delta g_{\alpha \beta} \cdot g^{c_{\alpha}}
\end{array}
$$

An important case of sample symmetry is the orthorhombic symmetry of rolled and recrystallized sheet. It contains 4 symmetry rotations $g^{s}$. An important case of crystal symmetry is the cubic one. It contains 24 symmetry rotations $g^{c}$. Hence, in the cubicorthorhombic case there are in general 96 symmetrically equivalent orientations $g_{\alpha}^{\prime}$. If both phases are cubic, then any misorientation 
between two crystals can in general be described by 576 symmetrically equivalent variants $\Delta g_{\alpha \beta}^{\prime}$.

The distribution functions must be invariant with respect to the crystal symmetries and to the sample symmetry. As far as the crystal symmetries are concerned, this is trivial since the choice of a specific crystal coordinate system among the crystallographically equivalent systems is only a matter of description which does not have any physical meaning. In the case of sample symmetry equivalence of two orientations means that these orientations occur in the sample with equal statistical frequency. The invariance of all distribution functions with respect to the sample symmetry operations can thus be considered as the definition of sample symmetry.

With the equivalences defined in Eqs. (22) and (23) the following invariance conditions are obtained

$$
\begin{gathered}
\phi^{\alpha \beta}\left(g^{c_{\beta}} \cdot \Delta g \cdot g^{c_{\alpha}}, g^{s} \cdot \mathbf{x}\right)=\phi^{\alpha \beta}(\Delta g, \mathbf{x}) \\
\psi^{\alpha \beta}\left(g^{c_{\beta}} \cdot \Delta g \cdot g^{c_{\alpha}}, g^{c_{\alpha}-1} \cdot \mathbf{k}\right)=\phi^{\alpha \beta}(\Delta g, \mathbf{k}) \\
F^{\alpha \beta}\left(g^{c_{\beta}} \cdot \Delta g \cdot g^{c_{\alpha}}\right)=F^{\alpha \beta}(\Delta g) \\
S^{\alpha \beta}\left(o^{s} \cdot \mathbf{x}\right)=S^{\alpha \beta}(\mathbf{x}) \\
\sigma^{\alpha \beta}\left(o^{c_{\alpha}} \cdot \mathbf{k}\right)=\sigma^{\alpha \beta}(\mathbf{k})
\end{gathered}
$$

\section{SPECIAL CASES}

It may be useful to consider some special cases of the distribution functions defined above. If misorientation distribution is independent of angular distribution of the boundaries then it is

$$
\begin{aligned}
& \phi^{\alpha \beta}(\Delta g, \mathbf{x})=F^{\alpha \beta}(\Delta g) \cdot S^{\alpha \beta}(\mathbf{x}) \\
& \psi^{\alpha \beta}(\Delta g, \mathbf{k})=F^{\alpha \beta}(\Delta g) \cdot \sigma^{\alpha \beta}(\mathbf{k})
\end{aligned}
$$

Furthermore, if the grains of the phases $\alpha$ and $\beta$ are assumed to be randomly distributed in space irrespective of their crystallographic orientation or shape then the misorientation function $F^{\alpha \beta}$ is completely determined by the respective textures (Bunge, Dahlem 1986, Plege 1987)

$$
F_{u}^{\alpha \beta}(\Delta g)=\int f^{\alpha}(g) \cdot f^{\beta}(\Delta g \cdot g) d g
$$


This function may be called the "uncorrelated" misorientation function. Indeed, Eq. (28) may be considered as the definition of random spacial distribution of the grains of the phases $\alpha$ and $\beta$. Hence, using this definition, the term "uncorrelated" is to be strictly kept apart from "random" misorientation which means independence of $F^{\alpha \beta}(\Delta g)$ of $\Delta g$, i.e.

$$
F_{\text {rand }}^{\alpha \beta}(\Delta g) \equiv 1
$$

If the physical misorientation function $F^{\alpha \beta}(\Delta g)$ deviates from the uncorrelated one, then we can define an orientation correlation function by

$$
\varphi^{\alpha \beta}(\Delta g)=\frac{F^{\alpha \beta}(\Delta g)}{F_{u}^{\alpha \beta}(\Delta g)}
$$

In this definition, orientation correlation means that certain orientation differences $\Delta g$ are preferredly assumed by $\alpha$ and $\beta$ crystals compared to their occurrence "by chance" which were to be expected by considering just the relative frequencies of crystal orientations $g^{\alpha}$ and $g^{\beta}$ i.e. the two textures. As an example, "random correlation"

$$
\varphi_{\text {rand }}^{\alpha \beta}(\Delta g) \equiv 1
$$

may be assumed in a primary recrystallized material immediately after impingement of the crystallites. Whereas a non-random correlation may develop gradually during continuous grain growth as a consequence of lower grain boundary energy of the boundaries of certain orientation differences e.g. the low- $\Sigma$ coincidence boundaries.

It should be mentioned that the uncorrelated misorientation distribution function for grain boundaries $F^{\alpha \alpha}(\Delta g)$ at the origin $\Delta g=\{0,0,0\}$ is identical with the texture index $J$ (see e.g. Bunge 1982)

$$
F_{u}^{\alpha \alpha}(0)=\int\left[f^{\alpha}(g)\right]^{2} d g=J^{\alpha}
$$

Furthermore, it is

$$
\int\left[f^{\alpha}(g)-f^{\alpha}(\Delta g \cdot g)\right]^{2} d g \geq 0
$$


from which follows

$$
\int\left[f^{\alpha}(g)\right]^{2} d g+\int\left[f^{\alpha}(\Delta g \cdot g)\right]^{2} d g \geq 2 \int f^{\alpha}(g) \cdot f^{\alpha}(\Delta g \cdot g) d g
$$

The two terms on the left-hand side are equal and, according to Eq. (32), they are equal to $J$. Hence, it is

$$
F_{u}^{\alpha \alpha}(\Delta g) \leq F_{u}^{\alpha \alpha}(0)=J^{\alpha}
$$

The uncorrelated misorientation distribution function always takes on its maximum value at the origin. Equation (35) does, however, not hold in general for the physical misorientation distribution $F^{\alpha \beta}(\Delta g)$ as it was defined in Eq. (12) including the case $F^{\alpha \alpha}(\Delta g)$.

\section{SERIES EXPANSION}

The functions $\phi$ and $\psi$ may be expanded into series in the following wav

$$
\begin{aligned}
& \phi^{\alpha \beta}(\Delta g, \mathbf{x})=\sum_{l=0}^{L} \sum_{\mu=1}^{M^{\alpha}(l)} \sum_{v=1}^{M^{\beta}(l)} \sum_{\lambda=0}^{\Lambda} \sum_{\sigma=1}^{N(\lambda)} A_{l \lambda}^{\mu \nu \sigma} \stackrel{i}{i}_{l}^{\mu \nu}(\Delta g) \dot{K}_{\lambda}^{\sigma}(\mathbf{x}) \\
& \psi^{\alpha \beta}(\Delta g, \mathbf{k})=\sum_{l=0}^{L} \sum_{\mu=1}^{M^{\alpha}(l)} \sum_{\nu=1}^{M^{\beta}(l)} \sum_{\lambda=0}^{\Lambda} \sum_{\rho=1}^{M^{\alpha}(\lambda)} B_{l \lambda}^{\mu \nu \rho} \dot{⿱ 亠 ⿻}_{l}^{\mu \nu}(\Delta g) \dot{K}_{\lambda}^{\rho}(\mathbf{k})
\end{aligned}
$$

where $\stackrel{i}{*}_{l}^{\mu v}(\Delta g)$ are symmetry invariant harmonic functions obeying the symmetry relationship Eq. (25). The functions $\dot{K}_{\lambda}^{\sigma}(\mathbf{x})$ are normalized spherical surface harmonics obeying the rotational sybsymmetry of the sample symmetry and $\overleftarrow{K}_{\lambda}^{\sigma}(\mathbf{k})$ are spherical harmonics of the crystal symmetry of the $\alpha$-phase. Similarly, the misorientation distribution functions are expanded in the form (Bunge 1982)

$$
F^{\alpha \beta}(\Delta g)=\sum_{l=0}^{L} \sum_{\mu=1}^{M^{\alpha}(l)} \sum_{\nu=1}^{M^{\beta}(l)} D_{l}^{\mu \nu} \stackrel{i}{i}_{l}^{\mu \nu}(\Delta g)
$$

The shape functions are written in the form (Bunge 1982)

$$
\begin{aligned}
& S^{\alpha \rho}(\mathbf{x})=\sum_{\lambda=0}^{\Lambda} \sum_{\sigma=1}^{N(\lambda)} E_{\lambda}^{\sigma} \dot{K}_{\lambda}^{\sigma}(\mathbf{x}) \\
& \sigma^{\alpha \beta}(\mathbf{k})=\sum_{\lambda=0}^{\Lambda} \sum_{\rho=1}^{M^{\alpha}(\lambda)} G_{\lambda}^{\rho} \dot{K}_{\lambda}^{\rho}(\mathbf{k})
\end{aligned}
$$


The dots on top of the functional symbols denote the sample symmetry $(\cdot)$, the $\alpha$-crystal symmetry (:), and the $\beta$-crystal symmetry ( $\vdots)$ respectively. Finally the textures are expanded in the form (Bunge 1969)

$$
f^{\alpha}(g)=\sum_{l=0}^{L} \sum_{\mu=1}^{M^{\alpha}(l)} \sum_{v=1}^{N(l)} C_{l}^{\mu v} \dot{T}_{l}^{\mu v}(g)
$$

By using symmetry invariant functions in Eqs. (36-39) the corresponding coefficients become linearly independent. Their variability range is then only restricted by the positivity condition of all distribution functions.

The texture index $J$ mentioned in Eq. (32) is expressed in the form

$$
J^{\alpha}=\sum_{l=0}^{L} \sum_{\mu=1}^{M(l)} \sum_{v=1}^{N(l)} \frac{\left[C_{l}^{\mu v}\right]^{2}}{2 l+1}
$$

With $K_{0}^{1}=\sqrt{4 \pi}$, Eq. (11) leads to

and Eq. (13) leads to

$$
D_{l}^{\mu \nu}=\sqrt{4 \pi} A_{l 0}^{\mu \nu 1}=\sqrt{4 \pi} B_{l 0}^{\mu \nu 1}
$$

$$
E_{\lambda}^{\sigma}=A_{0 \lambda}^{11 \sigma} ; \quad G_{\lambda}^{\rho}=B_{0 \lambda}^{11 \rho}
$$

Furthermore, in the special case Eq. (27), it is

$$
\begin{aligned}
& A_{l \lambda}^{\mu v \sigma}=D_{l}^{\mu \nu} \cdot E_{\lambda}^{\sigma} \\
& B_{l \lambda}^{\mu \nu \rho}=D_{l}^{\mu \nu} \cdot G_{\lambda}^{\rho}
\end{aligned}
$$

and the coefficients of the uncorrelatred misorientation distribution function Eq. (28) are expressed by those of the two texture functions (Ref. Bunge 1982)

$$
D_{l}^{\mu_{1} \mu_{2}}=\frac{1}{2 l+1} \sum_{v=1}^{N(l)} C_{l}^{\mu_{1} v}(\beta) \cdot C_{l}^{\mu_{2} v}(\alpha)
$$

The series expansion is a rather "economic" representation of distribution functions especially in highly symmetrical cases. In the cubic-cubic case for instance, the number of coefficients of each degree $l$ is given by $M(l)^{2}$ which is shown in Table 1 . Furthermore, the symmetry relation Eq. (19) applied to grain boundaries $\alpha \alpha$ leads to the condition

$$
D_{l}^{\mu v}=D_{l}^{\nu \mu}
$$


Table 1 Number of independent parameters of the series expansion of a misorientation distribution function in the case of cubic-cubic symmetry. $\alpha \beta=$ phase boundaries, $\alpha \alpha=$ grain boundaries, $n(l)=$ parameters of degree $l, N(l)=$ parameters of all degrees up to $l$

\begin{tabular}{rlrlllllrrrrrrrrrr}
\hline & $l$ & 4 & 6 & 8 & 9 & 10 & 12 & 13 & 14 & 15 & 16 & 17 & 18 & 19 & 20 & 21 & 22 \\
\hline$\alpha \beta$ & $n(l)$ & 1 & 1 & 1 & 1 & 1 & 4 & 1 & 1 & 1 & 4 & 1 & 4 & 1 & 4 & 4 & 4 \\
& $N(l)$ & 1 & 2 & 3 & 4 & 5 & 9 & 10 & 11 & 12 & 16 & 17 & 21 & 22 & 26 & 30 & 34 \\
\hline \multirow{2}{*}{$\alpha \alpha$} & $n(l)$ & 1 & 1 & 1 & 1 & 1 & 3 & 1 & 1 & 1 & 3 & 1 & 3 & 1 & 3 & 3 & 3 \\
& $N(l)$ & 1 & 2 & 3 & 4 & 5 & 8 & 9 & 10 & 11 & 14 & 15 & 18 & 19 & 22 & 25 & 28 \\
\hline
\end{tabular}

which further reduces the number of parameters as is also given in Table 1. It is seen from Table 1 that there is only one coefficient for each degree $l$ up to $l=10$. Hence, a cubic-cubic misorientation function is described by a total of 5 parameters up to $l=10$ (Bunge 1982). A rather good approximation of this function can thus be obtained by a small number of individual orientation measurements.

\section{MEASUREMENT BY ELECTRON DIFFRACTION}

At the present state of experimental technqiue, it seems to be virtually impossible to determine the boundary distribution functions Eq. (5) experimentally. Using electron diffraction, either with the back-reflection or transmission technique (Gotthard et al. 1973, Haessner 1981, Pospiech et al. 1986, Weiland and Schwarzer 1986), it is, however, possible to determine the two-dimensional analoga of the functions $\phi$ and $\psi$ and the deduced functions $F, S, \sigma$. The analogous functions $\phi_{(\mathbf{n})}$ and $\psi_{(\mathrm{n})}$ in a section perpendicular to the sample direction $\mathbf{n}$ are defined by

$$
\begin{aligned}
& \frac{d L^{\alpha \beta}\left(\Delta g, \mathbf{x}^{\prime}\right)}{L^{\alpha \beta}}=\phi_{(\mathbf{n})}^{\alpha \beta}\left(\Delta g, \mathbf{x}^{\prime}\right) d \Delta g d \Omega \\
& \frac{d L^{\alpha \beta}\left(\Delta g, \mathbf{k}^{\prime}\right)}{L^{\alpha \beta}}=\psi_{(\mathbf{m})}^{\alpha \beta}\left(\Delta g, \mathbf{k}^{\prime}\right) d \Delta g d \Omega
\end{aligned}
$$

where $L^{\alpha \beta}$ is the length of $\alpha \beta$ boundary lines in the considered planar section, $\mathbf{x}^{\prime}$ is the normal direction to the boundary line in the 
section and $\mathbf{k}^{\prime}$ is the crystallographic direction parallel to $\mathbf{x}^{\prime}$. Similarly, the misorientation function $F_{(\mathbf{n})}$ is defined by

$$
\frac{d L^{\alpha \beta}(\Delta g)}{L^{\alpha \beta}}=F_{(\mathbf{n})}^{\alpha \beta}(\Delta g) d \Delta g
$$

And finally also the texture functions may be defined twodimensionally

$$
\frac{d A^{\alpha}(g)}{A^{\alpha}}=f^{\alpha}(g) d g
$$

where now $A^{\alpha}$ is the total area of the $\alpha$-phase in the section. In a homogeneous sample the texture function defined according to Eq. (48) is identical with that of Eq. (14). Hence, the function in Eq. (48) has been written without an index (n). The other twodimensional functions, including $F_{(\mathbf{n})}^{\alpha \beta}$ may definitely depend on the orientation $\mathbf{n}$ of the section.

Furthermore, polycrystalline samples can often not be considered as being homogeneous. Then all the considered functions may depend on the location $\mathbf{r}$ within the sample at which they are being measured (see e.g. Bunge 1982b). In this case "local" and "global" quantities are to be distinguished. The global texture, for instance, is the integral over all local textures

$$
f^{\alpha}(g)=\int_{v} f^{\alpha}(g, \mathbf{r}) \mathrm{d} V
$$

where the integral is to be taken over the whole sample. Similar inhomogeneities are to be considered for all the other distribution functions.

As a general rule, the inhomogeneities will be the stronger, the smaller the considered volume elements. Hence, all functions determined by electron microscopy and electron diffraction must, in principle, be considered as local functions which may be different from sample to sample in the same material and which may also be different from the corresponding quantities determined by other methods e.g. the texture functions determined by $x$-ray or neutron diffraction. It must, however, also be admitted that, for instance, the correlation function $\varphi^{\alpha \beta}(\Delta g)$ may be found independent of the location $\mathbf{r}$ while the local textures $f^{\alpha}(g, \mathbf{r}) f^{\beta}(g, \mathbf{r})$ and the misorientation $F^{\alpha \beta}(\Delta g, \mathbf{r})$ are strongly $\mathbf{r}$-dependent (Plege 1986). 


\section{SINGLE ORIENTATION MEASUREMENTS}

Using electron microscopy and diffraction, the orientations $g_{i}^{\alpha}, g_{i}^{\beta}$ of the individual crystals $i, j$ of the phases $\alpha, \beta$ can be measured as well as the areas $A_{i}^{\alpha}, A_{j}^{\beta}$ and the boundary lengths $L_{i j}^{\alpha \beta}$ (Weiland, Schwarzer 1986). This allows one to determine the textures $f^{\alpha}(g)$, $f^{\beta}(g)$, the physical misorientation function $F^{\alpha \beta}(\Delta g)$ and the correlation function $\varphi^{\alpha \beta}(\Delta g)$. The textures and the misorientation function are defined by Eqs. (39) and (37) respectively with the coefficients

with

$$
C_{l}^{\mu v}=\frac{\sum_{i} A_{i} \cdot C_{l}^{\mu v}(i)}{\sum_{i} A_{i}}, \quad D_{l}^{\mu v}=\frac{\sum_{i j} L_{i j} \cdot D_{l}^{\mu v}(i j)}{\sum_{i j} L_{i j}}
$$

$$
C_{l}^{\mu v}(i)=a\left(l, \omega_{0}\right) \cdot \dot{T}_{l}^{\mu v}\left(g_{i}^{\alpha}\right) ; \quad D_{l}^{\mu v}(i j)=a\left(l, \omega_{0}\right) \cdot \dot{T}_{l}^{\mu \nu}\left(\Delta g_{i j}^{\alpha \beta}\right)
$$

where

$$
a\left(l, \omega_{0}\right)=\frac{\exp \left\{-\frac{1}{4} l^{2} \omega_{0}^{2}\right\}-\exp \left\{-\frac{1}{4}(l+1)^{2} \omega_{0}^{2}\right\}}{1-\exp \left\{-\frac{1}{4} \omega_{0}^{2}\right\}}
$$

is a "convergence factor" which depends on the spread $\omega_{0}$ of a Gaussian peak superimposed to each measured orientation $g_{i}$ or misorientation $\Delta g_{i j}$. The spread $\omega_{0}$ is to be chosen the smaller the higher the number of measured orientations $g_{i}$ or misorientations $\Delta g_{i j}$ (Wagner 1986).

It is to be mentioned that Eqs. (50-52) give the correct symmetries independent of which symmetry variant according to Eq. (23) is being used for $g_{i}$ or $\Delta g_{i j}$. This is due to the representation of the distribution functions by symmetry invariant harmonic functions. The symmetry invariance is an essential advantage of the representation of all orientation dependent distribution functions in the way described above. An other principle of dealing with symmetries has been used by Mackenzie (1964). He used the representation of orientations and misorientations by rotation axis and angle and selected the symmetry variant with the smallest rotation angle. This leads to non-constant distributions of the rotation axes as well as angles in the case of random distribution. Using this representation, it is much more difficult to 
estimate whether any deviation from random distribution is statistically significant or not (Prantl et al. 1987).

\section{BOUNDARY ENERGIES}

The energy of grain and phase boundaries depends on the misorientation $\Delta g$ as well as on the crystallographic boundary orientation $k$ whereas it must be independent of the spacial orientation $\mathbf{x}$ of the boundary. Hence, the total energy of $\alpha \beta$ boundaries is given by

$$
E^{\alpha \rho}=A^{\alpha \rho} \cdot \iint \gamma^{\alpha \beta}(\Delta g, \mathbf{k}) \cdot \psi^{\alpha \beta}(\Delta g, \mathbf{k}) d \Delta g d \Omega
$$

where $\gamma^{\alpha \beta}$ is the specific boundary energy. In the special case Eq. (27b), Eq. (53) can be split up into the two integrals

$$
E^{\alpha \rho}=A^{\alpha \beta} \cdot \int \bar{\gamma}^{\alpha \beta}(\Delta g) \cdot F^{\alpha \beta}(\Delta g) d \Delta g
$$

with

$$
\bar{\gamma}^{\alpha \beta}(\Delta g)=\int \gamma^{\alpha \beta}(\Delta g, \mathbf{k}) \cdot \sigma^{\alpha \beta}(\mathbf{k}) d \Omega
$$

In the case of single phase materials and if nearly equiaxed grains are being considered, then $\sigma^{\alpha \beta}(\mathbf{k})$ may be assumed to independent of $\mathbf{k}$. In this case, and if Eq. (27b) holds, the total grain boundary energy depends only on the misorientation function $F^{\alpha \beta}(\Delta g)$ as given in Eq. (54).

\section{SOME EXAMPLES}

The uncorrelated misorientation distribution function was defined in Eq. (28) as the "auto-convolution" of the texture function. It expresses itself most easily in terms of the coefficients $C_{l}^{\mu \nu}$ according to Eq. (44). This function was calculated for some different textures as is shown in Figures 3 to 6 . Figure $3 a$ is the texture of AlMn1 cold rolled $90 \%$. The corresponding uncorrelated MODF is given in Figure $3 \mathrm{~b}$. It is represented in $\varphi_{2}$-sections which are identical with $\varphi_{1}$-sections. Because of the symmetry, i.e. cubic in both coordinate 
systems, the represented range $0^{\circ} \leq \varphi_{1} \leq 90^{\circ}, 0 \leq \phi \leq 90^{\circ}, 0^{\circ} \leq \varphi_{2} \leq$ $45^{\circ}$ still contains 18 symmetry variants of each general $\Delta g$ (including $\left.\Delta g^{-1}\right)$. Figure $4 \mathrm{a}$ and b show the texture and MODF of the same material annealed 5 seconds at $600^{\circ} \mathrm{C}$ which corresponds to primary recrystallization and some continuous grain growth. Figure $5 \mathrm{a}$ and $\mathrm{b}$ are the texture and MODF of a low carbon steel cold rolled $90 \%$ and Figure 6, finally shows texture and MODF of the same steel after recrystallization $20 \mathrm{~h}$ at $560^{\circ} \mathrm{C}$.

Although the four textures are quite different, the differences in the MODFs are much smaller. This is not at all astonishing. Rather,
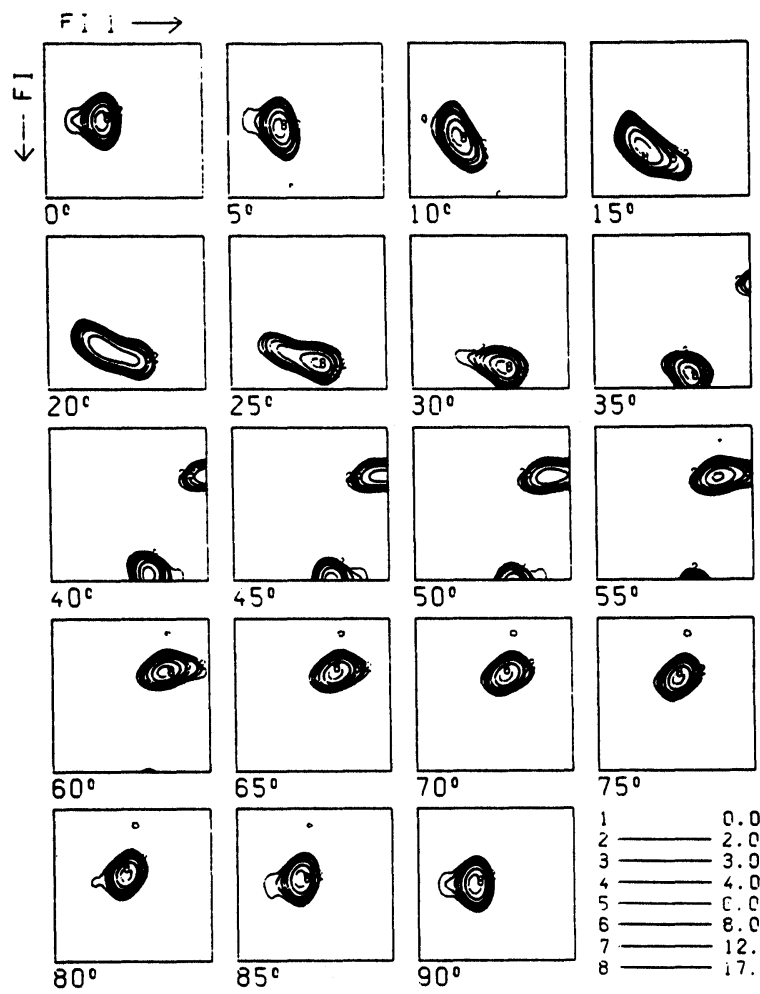

$35^{\circ}$
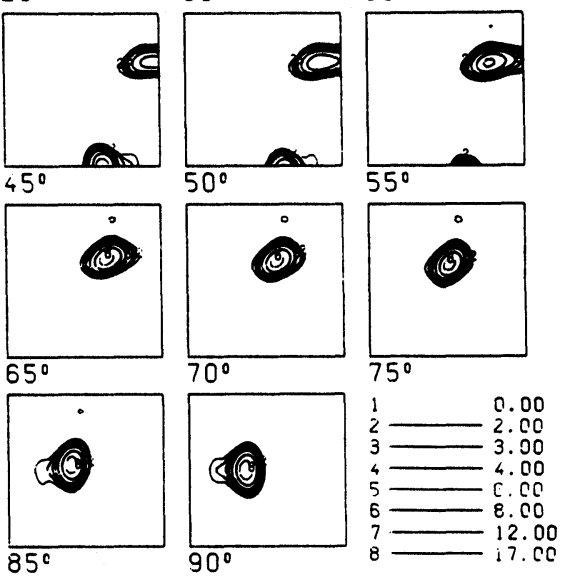

(a)

Figure 3 Texture and MODF of AlMn1 cold rolled 90\%. (a) the orientation distribution function ODF, (b) the uncorrelated misorientation distribution function MODF. 


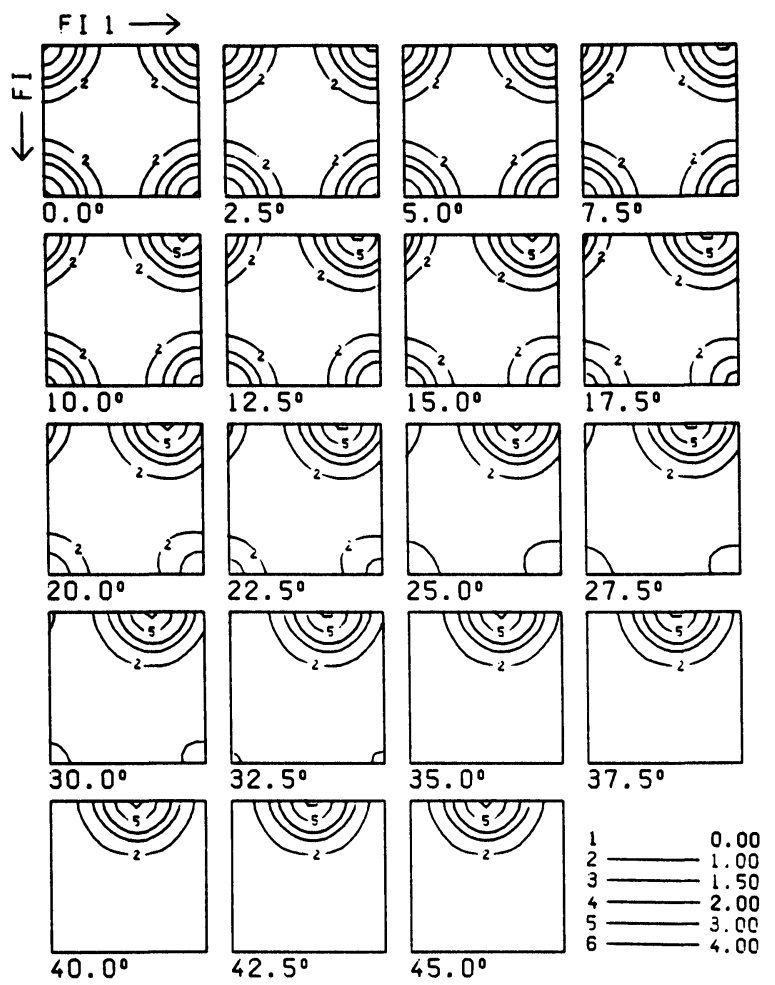

(b)

Figure 3b

it is due to the very definition of the uncorrelated MODF given in Eq. (28). According to Eq. (35), this function must take on its maximum value at $\Delta g=\{0,0,0\}$. Furthermore, the series expansion of this function must be very rapidly convergent. This can be seen from Eq. (44) expressing the coefficients in terms of those of the texture. The texture coefficients decrease with increasing $l$. Hence, the product of two such coefficients decreases even faster. Second, the terms in the sum in Eq. (44) may have opposite sign and finally also the factor $1 /(2 l+1)$ decreases with increasing $l$. Hence, the coefficients $D_{l}^{\mu_{1} / \mu_{2}}$ decrease much more rapidly than the coefficients $C_{l}^{\mu \nu}$ of the texture. This is shown for example in Figure 7 where the mean absolute values of these coefficients are plotted versus $l$ for the texture of Figure 3 as an example. Because of the 

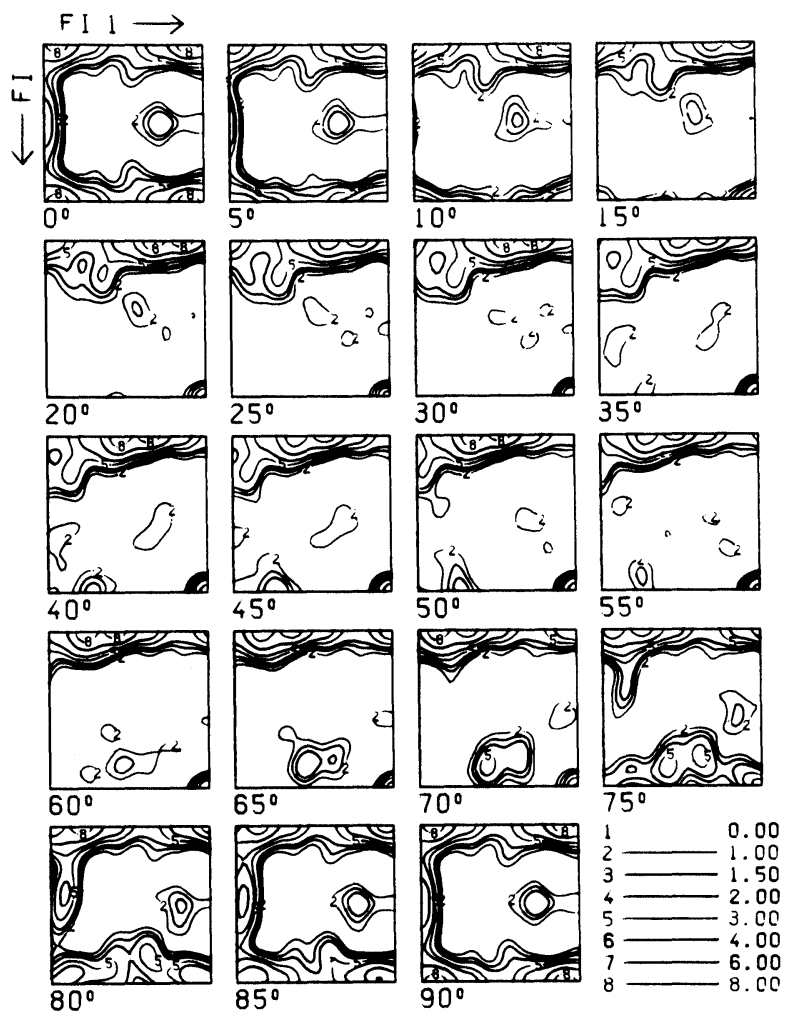

(a)

Figure 4 Texture and MODF of AlMn1 annealed 5 sec. at $600^{\circ} \mathrm{C}$ (a) ODF, (b) Uncorrelated MODF.

rapid convergence of the coefficients of the MODF, and bearing in mind the low number of linearly independent harmonic functions (Table 1), the MODF contains only a very low number of free parameters which explains the general similarity of the four MODFs. Nevertheless, there are differences in detail as is to be seen in Figure 8 which shows some sections of these functions through the $\Delta g$-space.

The physical MODF of recrystallized AlMn1 as defined according to Eq. (12) was also determined using individual orientation determination of neighbouring grain pairs in the transmission 


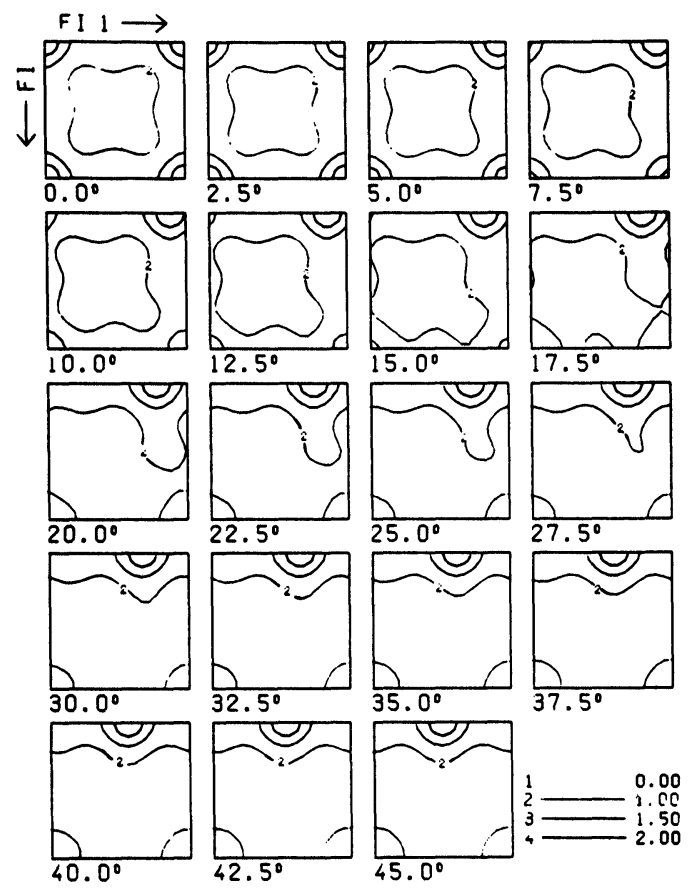

(b)

Figure 4b

electron microscope. The orientations were measured by means of Kikuchi lines (Weiland, Schwarzer 1986). In this way the orientation differences $\Delta g_{i j}^{\alpha \beta}$ of 144 pairs of grains were determined. From these values the MODF was obtained according to Eqs. (50-52) with $\omega_{0}=8^{\circ}$. The result is shown in Figure 9. This function is the "physical" MODF as compared with the "uncorrelated" MODF of this material shown in Figure 3b. Figure 9 is similar to Figure $3 b$ but there are differences in detail which can be seen best by calculating the correlation function $\varphi(\Delta g)$ defined in Eq. (30), i.e. by dividing the physical MODF by the uncorrelated one. The result is shown in Figure 10. It is seen that the actual frequency of grain boundary misorientations deviates from that expected according to the frequency of crystal orientations in the texture by a factor of 1.56 at the origin and it reveals a second peak at $\varphi_{1}=\phi=0^{\circ}$, $\varphi_{2}=45^{\circ}$. These two boundary misorientations are thus preferred 

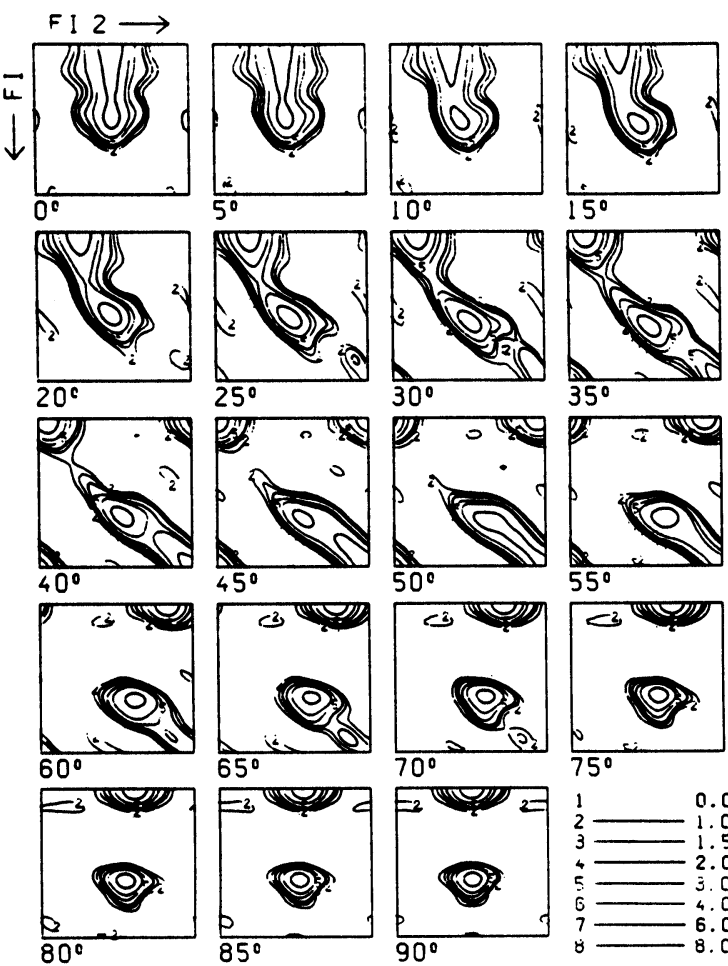

$55^{\circ}$
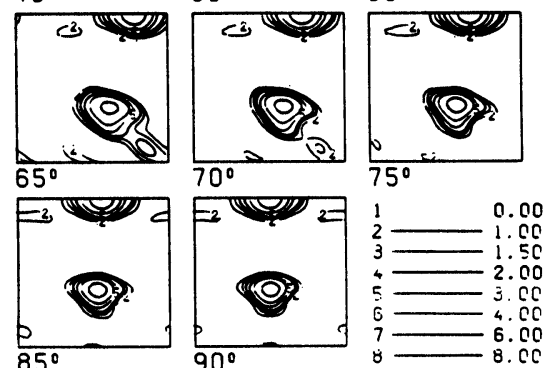

(a)

Figure 5 Texture and MODF of low carbon steel cold rolled 90\% (a) ODF, (b) Uncorrelated MODF.

from physical reasons with respect to the other ones. In the case of continuous grain growth, these reasons may be either a lower grain boundary energy or a higher mobility of these boundaries or both.

An example of two-phase correlation is given in Figure 11 which shows the misorientation distribution function between ferrite and martensite in a dual-phase steel deformed $14 \%$ in tension. The orientations $g_{i}^{\alpha}$ of ferrite and $g_{j}^{\beta}$ of neighbouring martensite grains were measured by means of Kikuchi lines (Weiland, Schwarzer, 1986). From these, the misorientations $\Delta g_{i j}^{\alpha \beta}$ of 49 neighbour pairs were obtained and the physical MODF was then calculated with $\omega_{0}=8^{\circ}$. The result is shown in Figure 11a. It is to be mentioned, that in this case the represented region contains 9 symmetry variants 

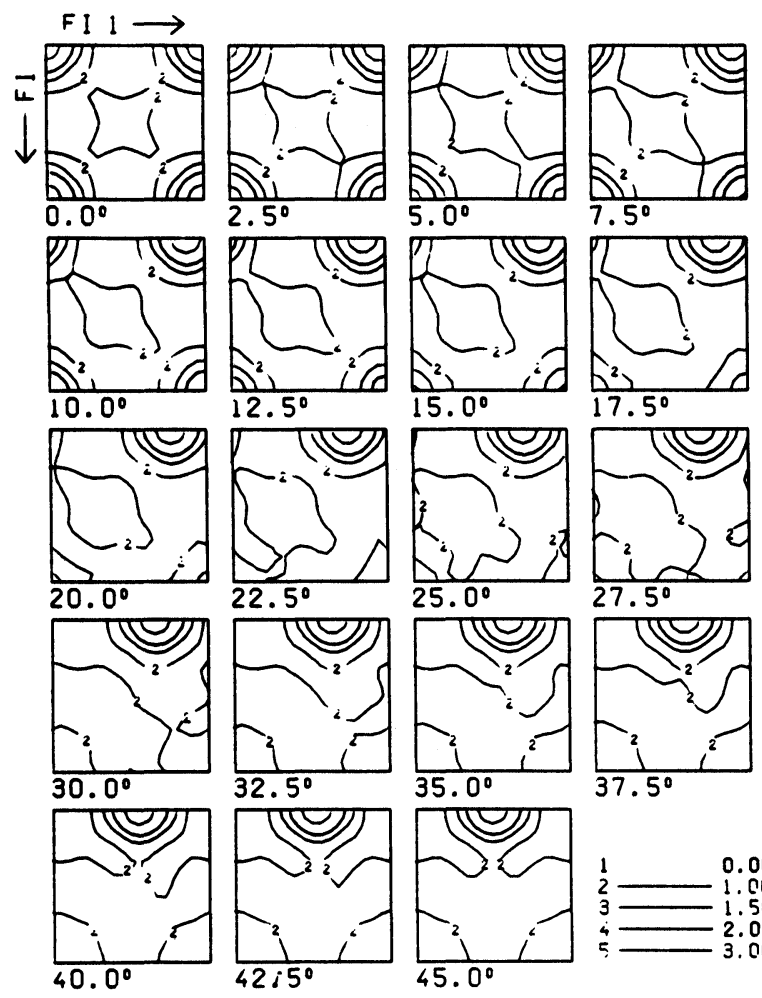

(b)

Figure 5b

for each general $\Delta g$ (in this case $\Delta g^{-1}$ is not symmetrically equivalent with $\Delta g$ ).

The uncorrelated MODF was obtained by selecting 300 pairs of $g_{i}^{\alpha}$ and $g_{j}^{\beta}$-values at random out of the above-mentioned ones. These pairs are generally not neighbours. The MODF calculated from the so obtained $\Delta g_{i j}^{\alpha \beta}$ is given in Figure $11 \mathrm{~b}$. It is the uncorrelated MODF as compared with the physical MODF of neighbour pairs given in Figure 11a. It is seen that the uncorrelated MODF is near to random whereas the physical MODF shows pronounced details. By dividing the function of Figure 11a by that of $11 \mathrm{~b}$ the correlation function $\varphi^{\alpha \beta}(\Delta g)$ is obtained which is shown in Figure 12. Since the uncorrelated MODF was very near to 

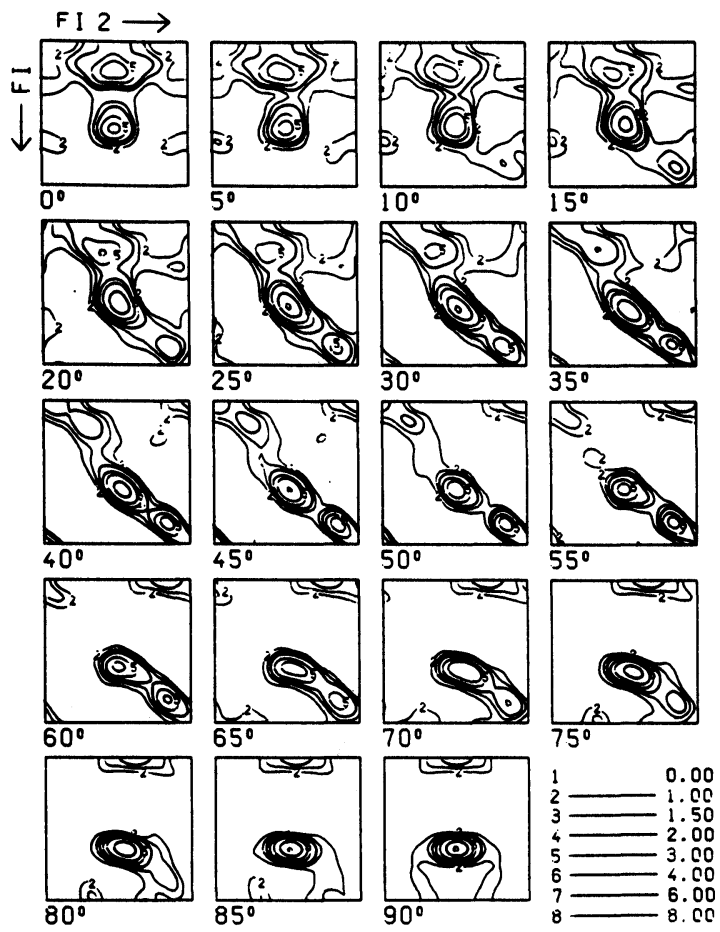

(a)

Figure 6 Texture and MODF of low carbon steel cold rolled $90 \%$ and annealed $20 \mathrm{~h}$ $560^{\circ} \mathrm{C}$. (a) ODF, (b) Uncorrelated MODF.

random in this case the correlation function $\varphi^{\alpha \beta}(\Delta g)$ is nearly identical with the physical MODF of Figure 11a. This result is, in some respect, opposite to that of the previous example in which the physical MODF was similar to the uncorrelated one. The two examples thus illustrate the two factors contributing to a physical MODF. A preference of certain misorientations $\Delta g$ in the grain or phase boundaries may arise simply because there are much more orientations in the texture which have just these misorientations. Hence, these misorientations are preferred by chance, simply from statistical reasons. This expresses itself in the uncorrelated MODF. This was mainly the case in the first example AlMn1. The second reason is a preference of certain misorientations $\Delta g$ from physical 


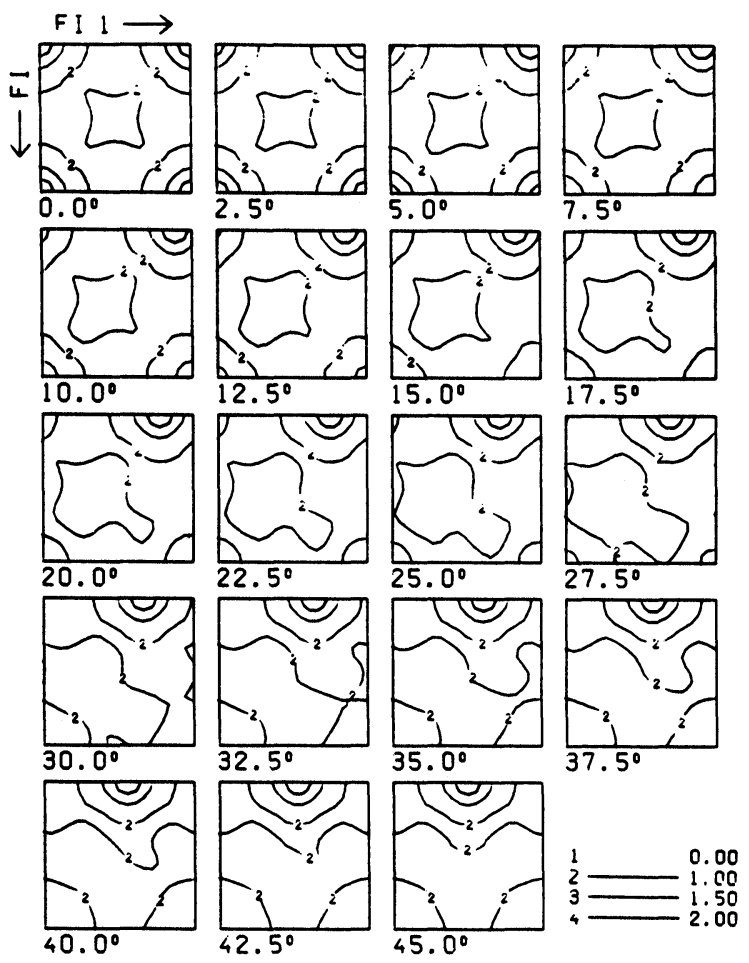

(b)

Figure 6b

reasons, e.g. grain boundary energy or a particular orientation relationship during phase transformation. This expresses itself most clearly in the correlation function $\varphi^{\alpha \beta}(\Delta g)$ which is thus cleared of the mere statistical effects. This is the main factor in the physical MODF of the second example.

In this second example, the uncorrelated MODF can also be obtained in a second way. First the ODFs of ferrite and martensite can be calculated separately from the corresponding $g_{i}^{\alpha}$ and $g_{j}^{\beta}$ (being the individuals of the above pairs). The so obtained ODFs are given in Figures 13a and b. The uncorrelated MODF is then obtained by the convolution of these two functions with each other according to Eq. (28) with the coefficients according to Eq. (44). The result of the "folding" is shown in Figure 14. It is similar to that 


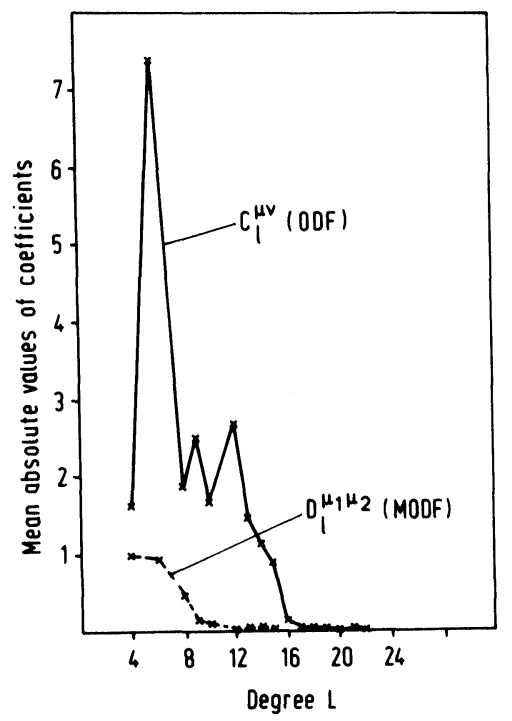

Figure 7 Mean absolute values of the coefficient of the $C_{l}^{\mu v}$ of the ODF and $D_{l}^{\dot{\mu}_{1} \mu_{2}}$ of the MODF for the texture of Figure 3.

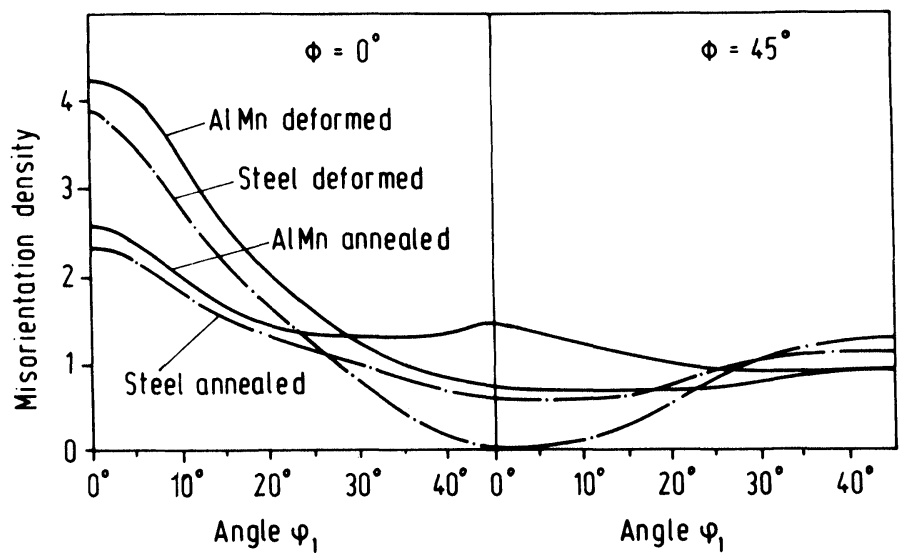

Figure 8 Sections through the MODFs of Figure 3 to 6 . (a) $\varphi_{1}=\phi=0^{\circ}$, (b) $\varphi_{1}=0^{\circ}, \phi=45^{\circ}$. 


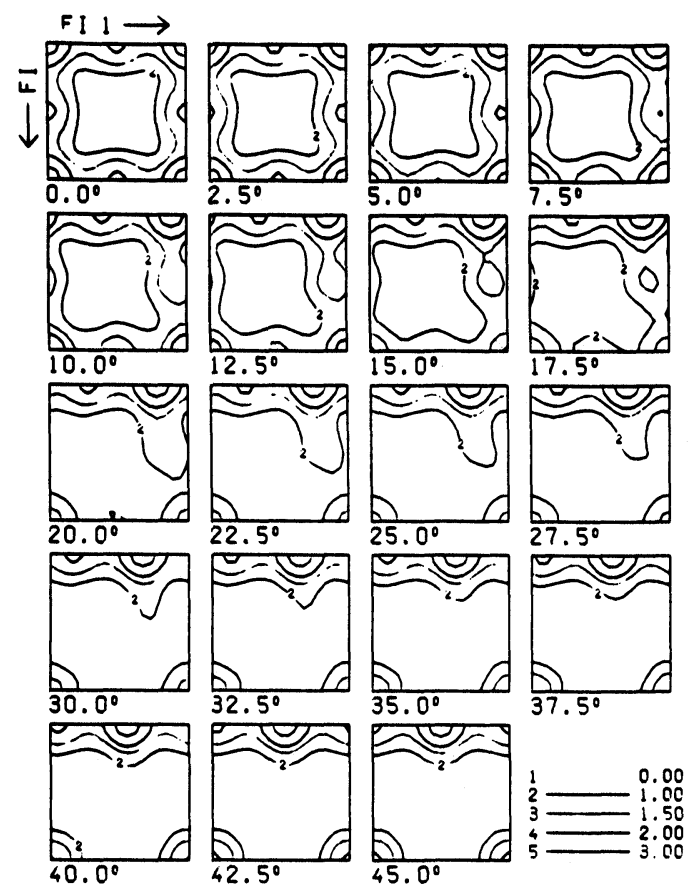

Figure 9 Physical MODF of AlMn1 cold rolled and recrystallized, obtained by electron diffraction measurement of $144 \Delta g_{i j}^{\alpha \beta}$-values of neighbouring crystal pairs (smoothed by $\omega_{0}=10^{\circ}$ ).

of Figure 11b. The differences between the two functions are due to the rather poor statistics obtained with 49 values each in Figures $13 \mathrm{a}, \mathrm{b}$ and 300 pairs in Figure 11b. In all experimental determinations of MODFs by individual orientation measurements the statistics is always the main problem. That has, indeed, two different reasons. First, the measurement of individual orientations is still a tedious task, notwithstanding a certain semiautomatization of the method (Weiland and Schwarzer, 1986). Hence, the number of individuals used in any statistical manifold is usually rather limited. The more severe reason is, however, to be seen in the inhomogenity of texture which is the stronger the smaller the scale at which texture is being considered. Hence, electron diffraction samples taken from different places of a macroscopic sample may 


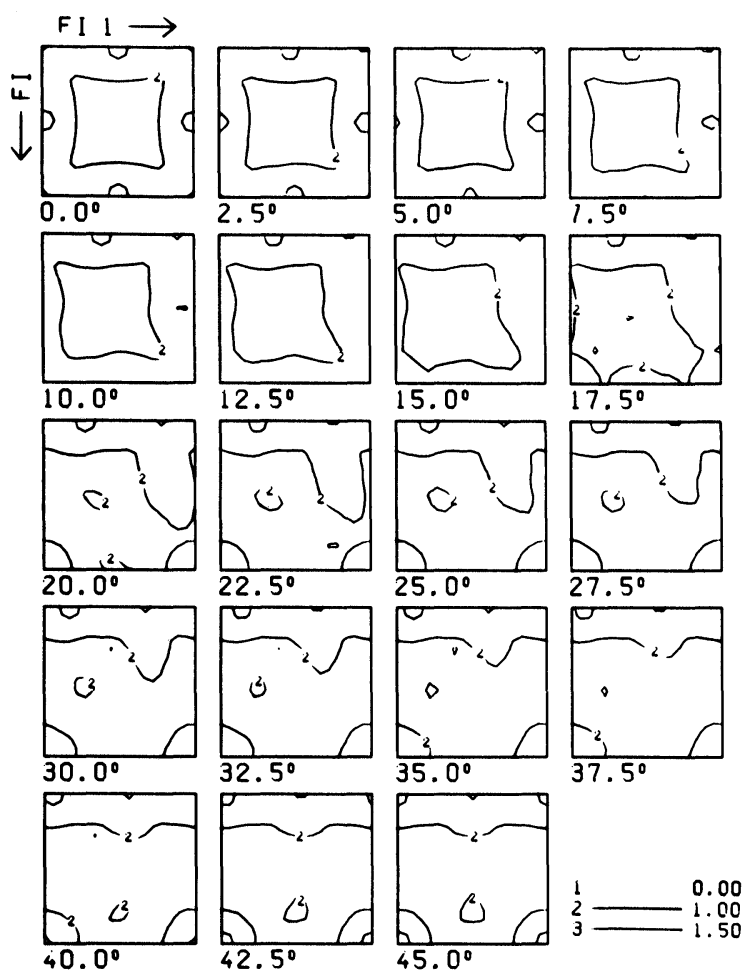

Figure 10 Orientation correlation function $\varphi(\Delta g)$ obtained by dividing the function of Figure 9 by that of Figure 3b.

show quite different local textures. In the present example of a dual phase steel it is not even possible to determine the global texture of the material (e.g. by $x$-rays or neutrons) since the Bragg angles of ferrite and martensite are too close to be separated. Hence, an $\mathrm{x}$-ray determination of the texture is always the weighted superposition of the ferrite and the martensite texture. In the present case, the sample contained about $20 \%$ martensite. Hence, the $x$-ray texture is "near" to the ferrite texture as is seen by comparing Figure 15 with the local ferrite texture of Figure 13a.

It was the purpose of the present paper to show how misorientation distribution functions and correlation functions can be determined and how they can be represented. It is not the purpose to 


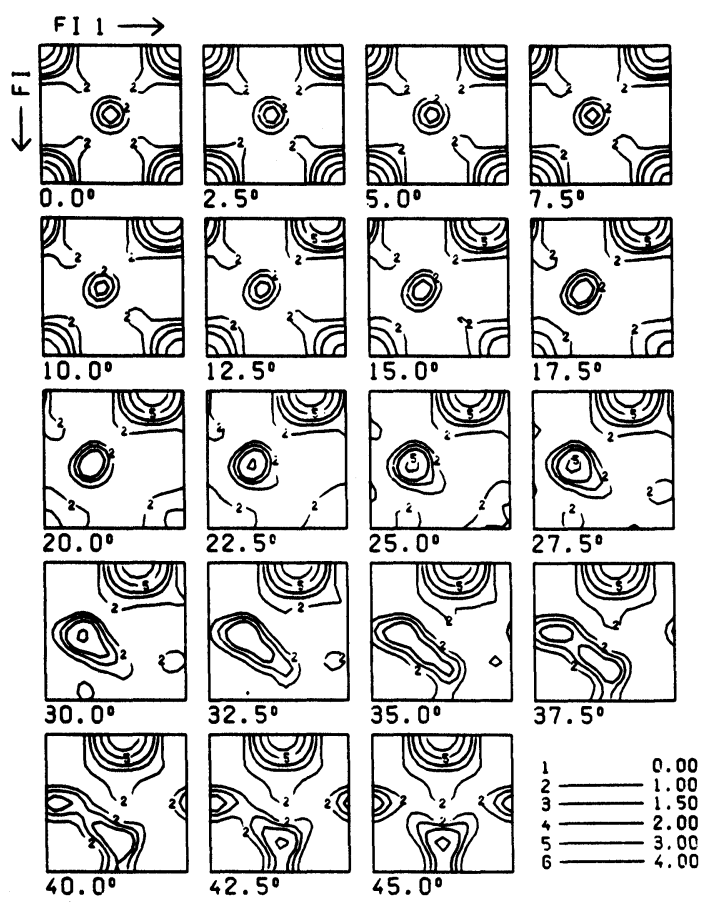

(a)

Figure 11 MODF of ferrite and martensite in a dual phase steel. (a) the physical MODF obtained from $\Delta g_{i j}^{\alpha \beta}$-values of 49 neighbour pairs measured by electron diffraction, (b) the uncorrelated MODF obtained from $\Delta g_{i j}^{\alpha \beta}$-values of 300 pairs selected at random among all ferrite orientations $g_{i}^{\alpha}$ and all martensite orientations $\boldsymbol{g}_{i}^{\boldsymbol{\beta}}$.

discuss the physical reasons leading to the specific functions given as examples. This will be done in other papers. It is then necessary to discuss the specific nature of boundaries the $\Delta g$-values of which correspond to peaks in the correlation function $\varphi^{\alpha \beta}(\Delta g)$. In this respect it is helpful to use "misorientation charts" which give the position of some specific misorientations $\Delta g$ in the Euler space. e.g. the Kurdjumov-Sachs or Nishiyama-Wassermann relationship or the various coincidence boundaries $\Sigma n$ (see e.g. Plege 1986). 


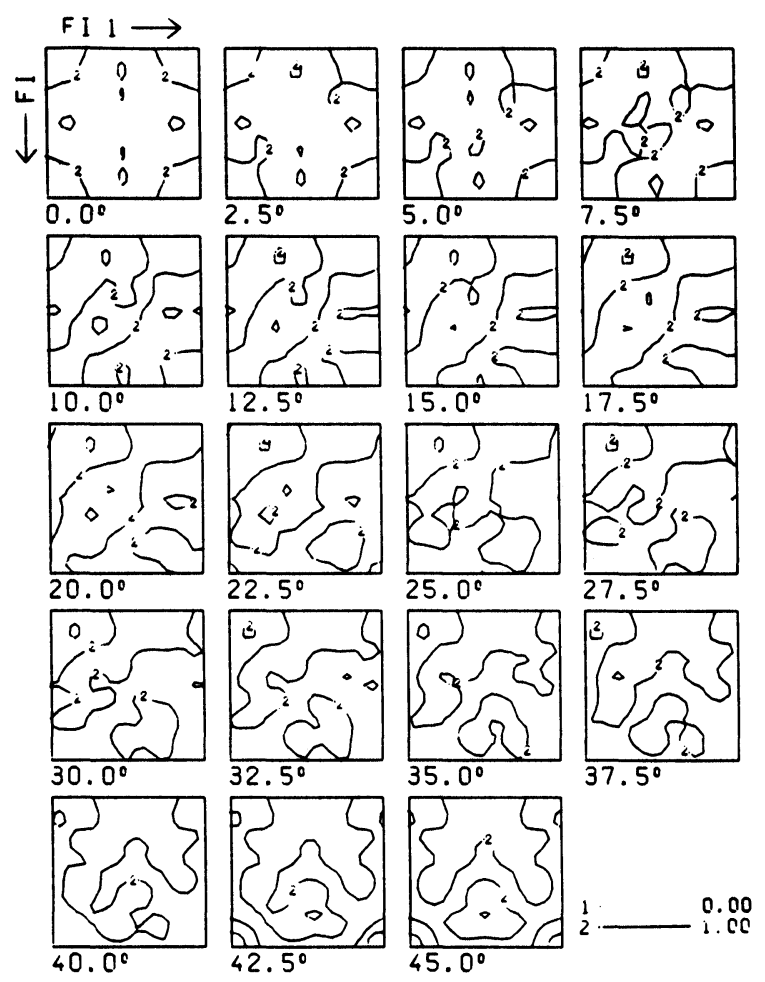

(b)

Figure 11b

\section{CONCLUSIONS}

In order to characterize grain and phase boundaries in polyphase materials, five angular parameters are needed. Three of them characterize the orientation difference across the boundary and two parameters characterize the boundary orientation either with respect to the macroscopic sample coordinate system or with respect to the crystal coordinate system of one of the neighbouring crystals. Hence, the grain boundary distribution is described alternatively by two five-dimensional boundary distribution functions.

If one considers only the dependence of the three misorientation 


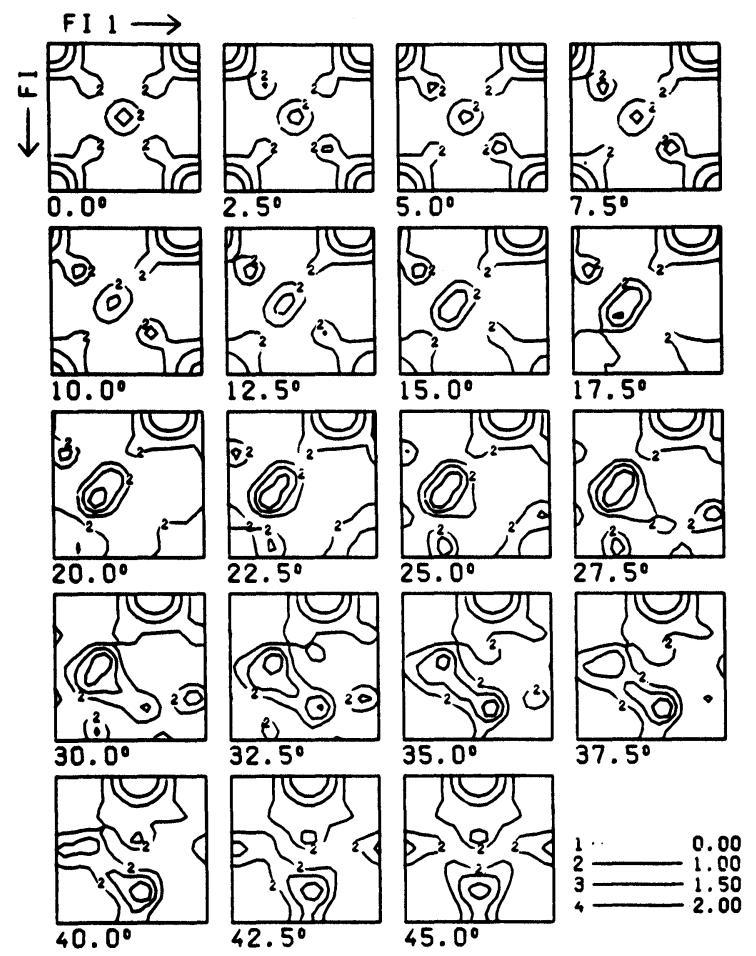

Figure 12 The correlation function $\varphi^{\alpha \beta}(\Delta g)$ for ferrite-martensite grain boundaries obtained by dividing the function Figure 11a by that of Figure 11b.

parameters $(\Delta g)$ then a misorientation distribution function (MODF) is obtained.

If one considers only the boundary orientation then two orientation or "shape" functions are obtained.

The functions must obey the crystal symmetries of the involved phases and the sample symmetry.

The functions can be represented by series expansion which is especially "economic" in the case of high symmetries e.g. cubic symmetries.

Two-dimensional analogae of the general distribution functions can be obtained from individual orientation measurements e.g. by Kikuchi lines in the transmission electron microscope. 


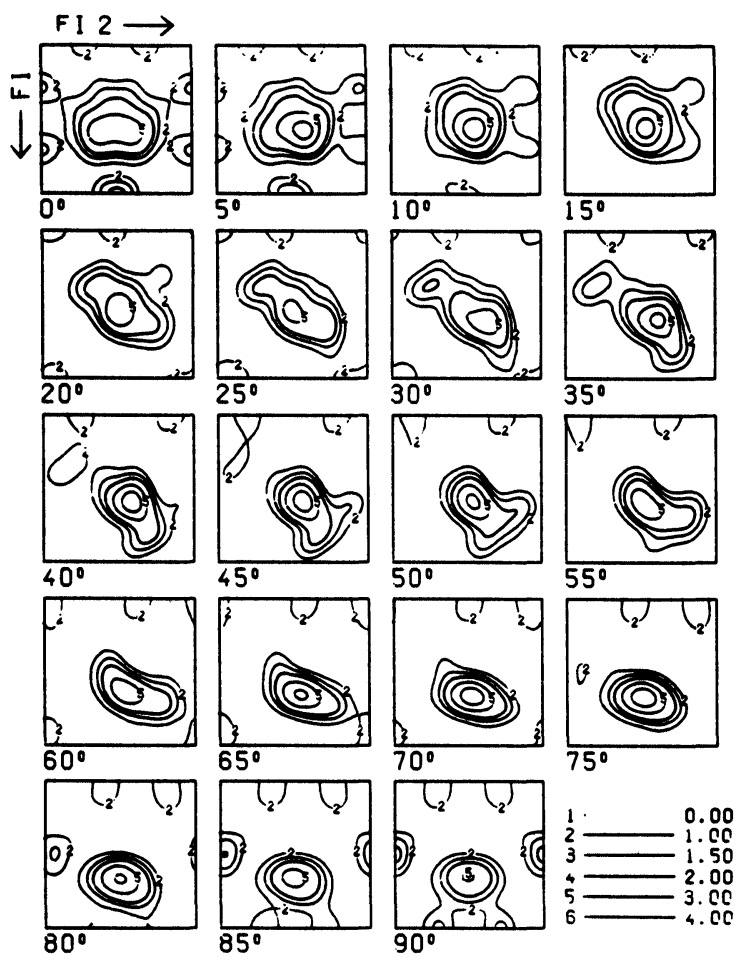

(a)

Figure 13 The textures of ferrite and martensite ina dual phase steel obtained by individual orientation measurement in the electron microscope. (a) Ferrite (110 orientations $g_{i}^{\alpha}, \omega_{1}=8^{\circ}$ ), (b) Martensite (203 orientations $g_{j}^{\beta}, \omega_{1}=8^{\circ}$ ).

As a special case, the "uncorrelated" misorientation distribution function is considered. It is defined on the basis of the textures of the involved phases irrespective of the neighbourship relations of the considered crystals.

The "physical" misorientation distribution function of grain or phase boundaries may be divided by the "uncorrelated" one deduced from the textures. The so obtained function was called the "correlation function". It describes the preference for certain grain or phase boundary misorientations $\Delta g$ from "physical reasons" (e.g. energetical reasons or orientation relationships due to oriented phase transformation). 


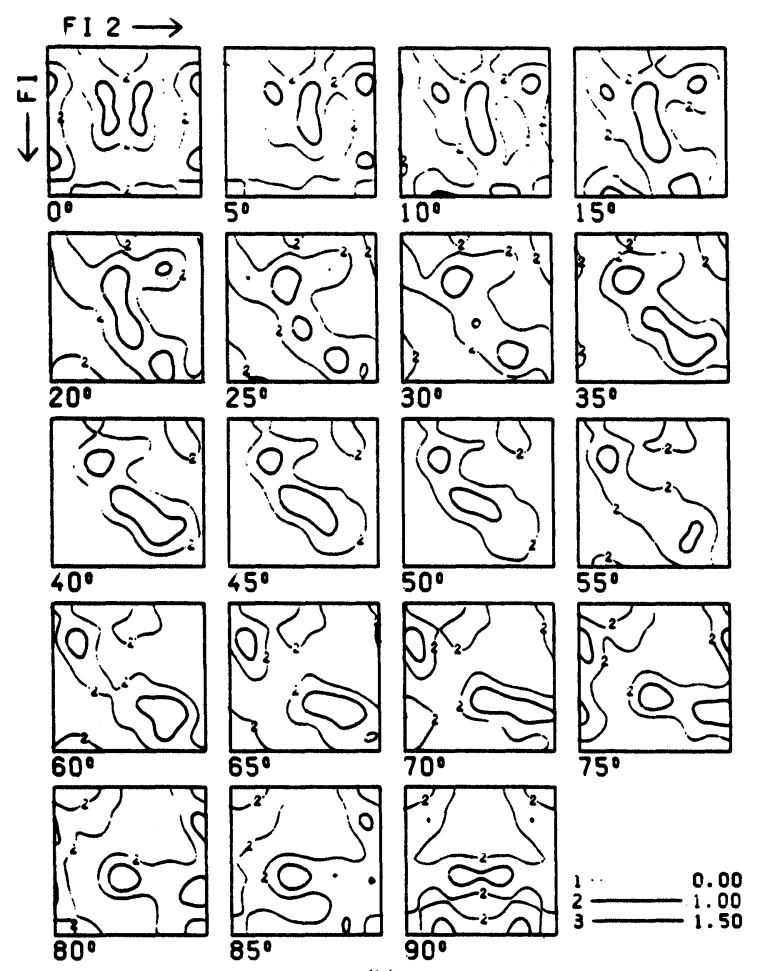

(b)

Figure 13b

The uncorrelated MODFs always have their maximum value at $\Delta g=0$, this is in the region of small angle grain boundaries. Furthermore, the series expansions of these functions converge very rapidly. Hence, the angular variation of these functions in the $\Delta g$-space is not very rapid. In the case of high crystal symmetry (e.g. cubic in both phases) the asymmetric unit in the $\Delta g$-space is rather small. Together with the slow angular variation of the uncorrelated MODFs these functions do not reveal much detail. Rather, they are very similar even for rather different textures as was shown in several examples. This behaviour is, however, not astonishing. Rather, it is due to the definition of the uncorrelated MODF. Within the usual spread ranges of textures a wide range of $\Delta g$-values can always be found. And if one bears in mind that in 


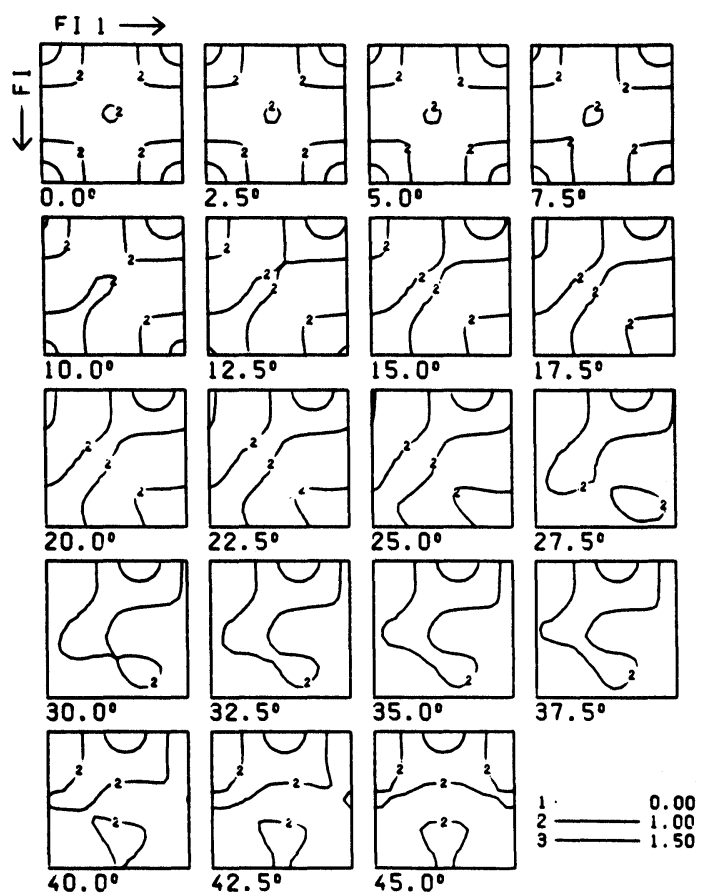

Figure 14 Uncorrelated MODF obtained from the textures Figures 13a and b.

the case of high crystal symmetries all symmetrically equivalent values are superposed in the $\Delta g$-space, then it is evident that the uncorrelated MODFs of different textures may look very similar as it was really found.

The uncorrelated MODF is the expectation value of any orientation difference $\Delta g$ if the grains of the textured material were distributed at random in space such that they become neighbours simply according to their relative frequency. A situation like that may be expected, for example, immediately after primary recrystallization, assuming that the nucleation sites are not correlated to each other.

If there is some physical preference for certain grain or phase boundary orientation differences $\Delta g$ and if the material is textured then the uncorrelated MODF is, so to speak, the basic level by which any MODF has to be divided in order to "sieve out" the 


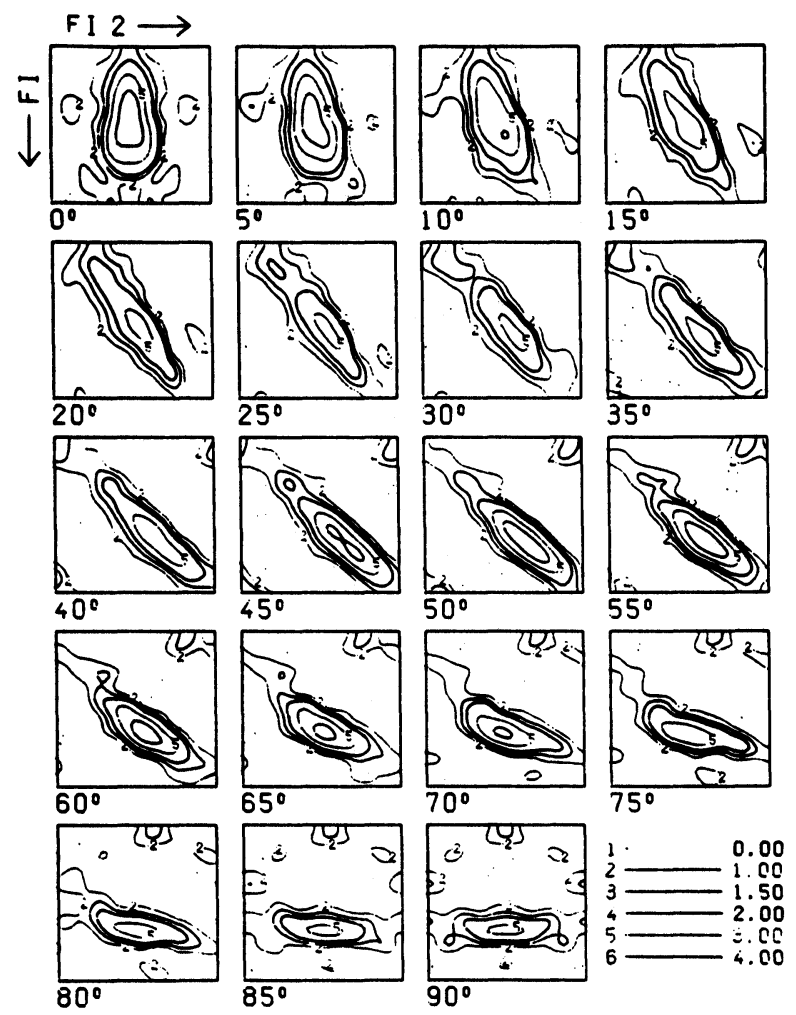

Figure 15 Texture of the dual phase steel obtained by neutron diffraction to be compared with the ferrite texture Figure 13a.

physical preferrence, which may be due to lower boundary energy in the course of grain coarsening processes or due to specific orientation relationships during phase transformation. These physical effects are then best seen in the deviation of the correlation function $\varphi^{\alpha \beta}(\Delta g)$ from unity.

\section{Acknowledgment}

This work was sponsored by the German Minister of Research and Technology BMFT under contract S 339-0. 


\section{References}

Adams, B. L. (1986). Description of Intercrystalline Structure Distribution in Polycrystalline Materials. Met. Trans A 17, 2199-2207.

Bunge, H. J. (1969). Mathematische Methoden der Texturanalyse. Akademie-Verlag Berlin.

Bunge, H. J. (1982). Texture Analysis in Materials Science, Butterworths Publishers London.

Bunge, H. J. (1982). Inhomogene Texturen, Z. Metallkde. 73, 483-488.

Bunge, H. J. and Esling, C. (1985). Symmetries in Texture Analysis. Acta Cryst. A41, 59-67.

Bunge, H. J. and Dahlem, E. (1986). Texture changes and growth anomalies during continuous grain growth. In: Annealing Processes, Recovery, Recrystallization and Grain Growth, Eds. N. Hansen, D. Juul-Jensen, T. Lefters, B. Ralph, Ris $\varnothing$. p. 255-260.

Gotthard, R., Hosche, G., Reimold, O. and Haessner, F. (1973). Topographic arrangement of crystallite orientations in rolled copper. Texture 1, 99-109.

Haessner, F. (1981). Orientation topography and its analysis in rolled material. Proc. ICOTOM 6 Tokyo p. 223-238.

Hilliard, J. E. (1962). Trans. Met. Soc. AIME. 224, 1201-1211.

Mackenzie, J. K. (1964). Acta Met. 12, 223-225.

Plege, B. (1986). Determination of orientation correlation functions in two-phase fibre composites. In: Experimental Techniques of Texture Analysis. Ed. H. J. Bunge. DGM Informationsgesellschaft Oberursel 315-328.

Plege, B. (1987) On Orientation Correlations and their Relationship to Textures. In: Theoretical Methods of Texture Analysis, DGM Informationsgesellschaft Oberursel, Ed. H. J. Bunge p. 393-403.

Pospiech, J., Sztwiertnia, K. and Haessner, F. (1986) The misorientation distribution function. Textures and Microstructures 6, 201-215.

Prantl, W., Werner, E. and Stüwe, H. P. (1987). Statistical treatment of measured orientation relationships. Textures and Microstructures (in print).

Wagner, F. (1986). Texture determination by individual orientation measurements. In: Experimental Techniques of Texture Analysis, Ed. H. J. Bunge. DGM Informationsgesellschaft Oberursel p. 115-123.

Weiland, H., Schwarzer, R. (1986) On Line texture determination by Kikuchi or channeling patterns. In: Experimental Techniques of Texture Analysis. Ed. H. J. Bunge. DGM Informationsgesellschaft Oberursel p. 301-313.

Zhao, J., Adams, B. L. and Morris, P. R. (1987) A comparison of measured and texture-estimated misorientation distributions in type 304 stainless steel tubing. Textures and Microstrauctures (in print). 